\title{
After Failure Repair Optimization in Dynamic Flexgrid Optical Networks
}

\author{
M. Ruiz ${ }^{1 *}$, M. Żotkiewicz ${ }^{2}$, A. Castro ${ }^{1}$, M. Klinkowski ${ }^{3}$, L. Velasco ${ }^{1}$, M. Pióro ${ }^{2}$ \\ 1 Optical Communications Group (GCO), Universitat Politècnica de Catalunya (UPC), Barcelona, Spain \\ 2 Institute of Telecommunications, Warsaw University of Technology (WUT), Warsaw, Poland \\ 3 Dept. Transmission and Optical Technologies, National Institute of Telecommunications (NIT), Warsaw, Poland \\ *e-mail:mruiz@ac.upc.edu
}

Abstract: We introduce the problem of reoptimizing the network after a link failure has been repaired as an effective way for reducing capacity usage and improving network performance. Numerical results show gains higher than $42 \%$.

(C) 2013 Optical Society of America

OCIS codes: (060.0060) Fiber optics and optical communications; (060.4250) Networks

\section{Introduction}

The advances in flexgrid optical technology [1] achieved in the last few years make it possible to deal with short-term and long-term traffic expectations. Such features as fine spectrum granularity (a result of dividing the spectrum into narrow frequency slices, e.g., $6.25 \mathrm{GHz}$ ), advanced optical modulation formats, and filtering and signal processing, constitute the basis for introducing flexibility and elasticity to network operation [2].

In the context of dynamic networks, a centralized active stateful Path Computation Element (PCE) can be used to deal with dynamic operations which include solving the Routing and Spectrum Assignment (RSA) problem for incoming connection requests [3], network reoptimization concerning spectrum defragmentation [2], and lightpath restoration to overcome network failures [4].

In case of a link failure, the use of bulk path computation was recently proposed to increase the amount of restored lightpaths without dramatically increasing restoration times, which are kept in the order of hundreds of ms [4]. Note that between the restoration instant and the failure repair instant, the network operates with reduced available capacity, thus increasing the blocking probability of new connection requests. Once the link is repaired, the overall available capacity of the network increases. However, the difference in terms of free available capacity between the highest and the lowest loaded links becomes a problem when solving the RSA for new connection requests. This is caused by the difficulty of finding routes with a sufficient number of continuous and contiguous slices and, therefore, the blocking probability remains high after the failure repair.

In this paper, we focus on the problem of reoptimizing the network after a failure repair (hereafter, After Failure Repair Optimization, AFRO) with the objective of improving network performance. In order to attain a globally optimized network, AFRO uses bulk path computation to find, for some of the existing lightpaths, new routes using the recently repaired link.

\section{After Failure Repair Optimization}

In order to illustrate the application of AFRO, we show in Fig. 1 an evolution of a dynamic network subject to a certain link failure. Moreover, in Fig. 2, which complements Fig. 1, we show an illustrative example of the network state at different time instants. Here, we consider a dynamic network that serves a set of lightpaths under normal operation (network state $t 0$ ). After an event of a link failure, the restoration mechanism finds an alternative route $(t 1)$ for each of the lightpaths affected by the fiber cut. Specifically, Fig. 2a shows a network with three lightpaths, namely $p 1, p 2$, and $p 3$, just before the event of a fiber cut in link 2-7. After that, lightpaths $p 1$ and $p 2$ are rerouted by the restoration algorithm (Fig. $2 \mathrm{~b}$ ).

Once the solution of the restoration algorithm is implemented, the network operates without the failed link until the failure is repaired (6-12 hours are usually needed to repair a fiber link). At this point, we can see that when repairing the link, and hence increasing the network capacity, the network performance is not necessarily improved. Namely, in Fig. $2 \mathrm{~b}$ one can see that a new connection requesting 4 slices between nodes 1 and 6 cannot be established when the link is broken and when the capacity of the repaired link is available again.

As previously stated, the network is reoptimized after the link is repaired $(t 2)$ by solving AFRO. Note that this reoptimization needs to be coordinated with other operations and properly scheduled, so that AFRO is not applied immediately after the failure repair. Moreover, the time needed for solving AFRO and implementing network changes must be short in order to reduce the delay of establishing new connection requests; note that this process is stopped during the AFRO processing time. After solving AFRO and implementing 


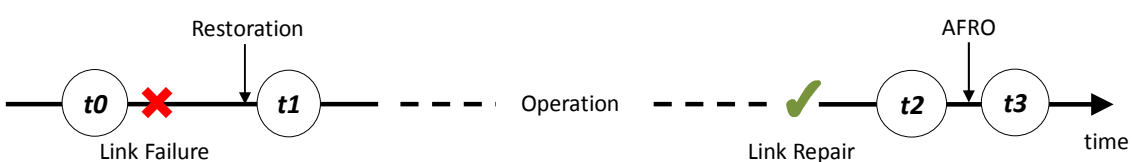

Fig. 1. Network states $(t)$ in time
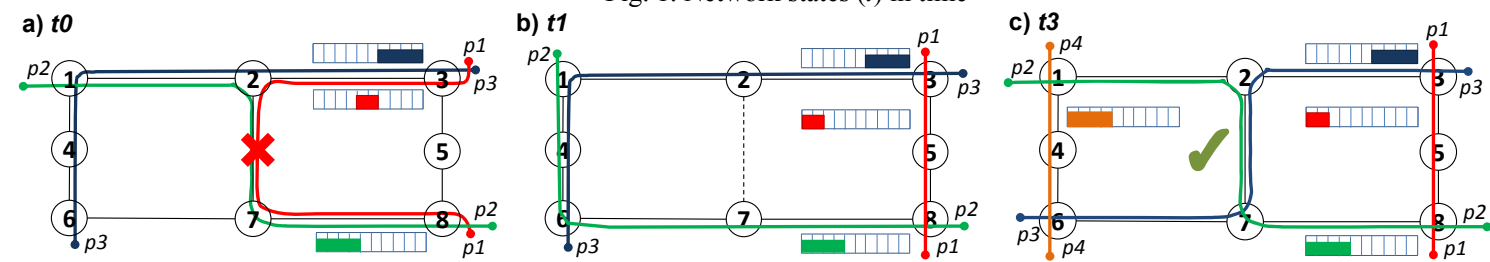

Fig. 2. Illustrative example

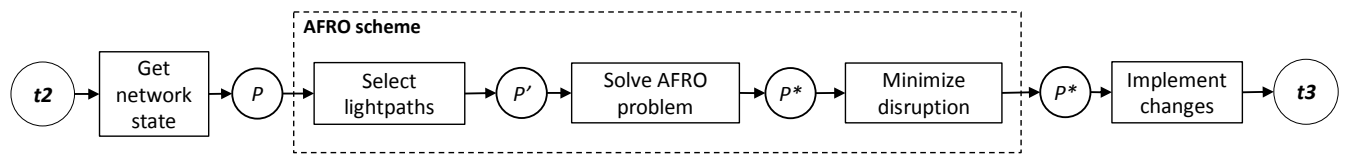

Fig. 3. AFRO scheme

its solution ( $t 3)$, the network is ready to operate and its performance is expected to be improved. As can be seen, the connection request between 1 and 6 can now be established (p4).

The scheme to apply AFRO is proposed in Fig. 3. Starting from the set of lightpaths established in $t 2(P)$, some of them are selected for reoptimization. Specifically, those lightpaths whose current route is longer than the shortest route crossing the repaired link are selected $\left(P^{\prime}\right)$. Next, the AFRO problem is solved and the reoptimized set of lightpaths $\left(P^{*}\right)$ is obtained. Finally, the solution is arranged in a sequence to minimize disruptions during lightpath rerouting.

Finally, the AFRO problem can be stated as follows:

Given: a) a network topology $G(N, E)$ defined as a set of optical nodes $N$ and a set of fiber links $E$; b) an optical spectrum divided into frequency slices of a given width; c) the set of slices used by non-selected lightpaths;

d) a set of lightpaths selected for reoptimization $P^{\prime}$; and e) a link $e^{r} \in E$, representing the link recently repaired. Find: the route and spectrum allocation for every lightpath in $P^{*}$.

Objective: minimize the total number of used slices so that every lightpath in $P^{*}$ has a new route containing $e^{r}$.

In order to effectively solve AFRO for real networks with hundreds of lightpaths, we have designed a specific column generation algorithm based on the work recently proposed in [6]. In short, the column generation algorithm allows near-optimal solving Mixed Integer Programming (MIP) formulations for the RSAbased problems in an affordable way, by considering a reduced set of problem variables (corresponding to the lightpaths) without significantly affecting the quality of the obtained solution. In the following section, both the AFRO performance and the efficiency of the implemented algorithm are evaluated for large network instances.

\section{Illustrative Numerical Results}

To evaluate the performance of AFRO, we used two nation-wide core topologies, namely, the 22-node British Telecom (BT) and the 30-node Telefonica (TEL) networks. We considered the fiber links with the spectrum width equal to $4 \mathrm{THz}$, divided into frequency slices of $6.25 \mathrm{GHz}$ (640 slices per link). Simulations were performed by means of an ad-hoc event-driven simulator implemented in OMNET++ [3]. Connection requests were generated following a Poisson process and lightpaths were torn down after an exponentially distributed holding time. The source and destination nodes were uniformly chosen and the requested bitrate was uniformly selected between values 100 and $400 \mathrm{~Gb} / \mathrm{s}$. Assuming QPSK as modulation format (whose spectrum efficiency is $2 \mathrm{bit} / \mathrm{s} / \mathrm{Hz}$ ), the number of slices requested for each connection is 8 and 32 slices, respectively.

For each topology, a set of randomly generated instances were obtained as follows: first, traffic was generated until reaching a steady state close to $1 \%$ of blocking probability. Then, a network link was randomly chosen and marked as failed. To restore the lightpaths affected by the link failure, the bulk restoration algorithm described in [4] was used aiming at restoring $100 \%$ of failed lightpaths. After implementing the solution of the restoration algorithm, the AFRO scheme was applied and its solution was implemented in the network.

Table 1 contains the main AFRO performance results for the above described instances (average values are shown). As can be observed, AFRO finds a new route for 15 and 27 lightpaths on the average for BT and TEL, respectively. This represents a significant proportion of improved lightpaths (around $42 \%$ of the selected ones). To obtain such solutions, the column generation algorithm needs just below 70 seconds. Assuming that the additional time for implementing the solution is smaller than 5 seconds (i.e., 50 sequential lightpath setups of $100 \mathrm{~ms}$ ), the total reoptimization time is small enough to ensure that during the reoptimization process only $0.1 \%$ of new connection requests will be processed with a delay not higher than 1 minute for an inter-arrival time as short as 2 hours. 
To evaluate the solutions obtained by AFRO, we compare columns $t 0, t 2$, and $t 3$ in Table 1 . For the sake of simplicity, we assume that the network after restoration remains unchanged until AFRO is applied (i.e., $t 1=t 2$ ). As shown, AFRO improves the network capacity usage since both the average number of used slices per link and the load of the most loaded link are smaller in $t 3$ than in $t 2$. However, this improvement is not observed when comparing $t 0$ and $t 3$ where link usage is practically the same. Moreover, $t 3$ contains links with a higher number of used slices than $t 1$.

Aiming at evaluating the network performance at different states, in Fig. 4 we show the connection blocking probability (weighted by connections bitrate) as a function of the traffic load (normalized with respect to the highest value). In view of the figures, solving AFRO increases the amount of new traffic that can be supported at $1 \%$ of blocking probability ( $55 \%$ for BT, and 108\% for TEL). Clearly, AFRO improves the performance of a network after a failure repair. Moreover, remarkable gains (above 40\%) with respect to $t 0$ are also obtained.

For a finer analysis of the available free capacity in $t 0$ and $t 3$, the fragmentation of the free spectrum was computed by means of the Shannon link entropy formula proposed in [7]. This entropy value is lower when the fragmentation of free spectrum decreases. Note that a less fragmented spectrum leads to a smaller blocking probability in the dynamic operation. In light of the link entropy results in Table 1, we can conclude that the spectrum in $t 3$ is less fragmented (lower entropy values) than in $t 1$ (higher entropy values), and, therefore, this is the rationale behind the differences observed in the blocking probability analysis.

Eventually, we performed the post-processing of AFRO solutions to minimize the traffic disruption when migrating from $t 2$ to $t 3$. To this aim, we computed the number of lightpaths that could be moved to new routes with a hitless rerouting, i.e., with a make-before-break (MBB) strategy. Results are depicted in Fig. 5 for both networks and different number of lightpaths to reroute. As shown, the percentage of lightpaths that can be hitless rerouted by means of $\mathrm{MBB}$ varies between $83 \%$ and $95 \%$. Moreover, the amount of lightpaths that should be rerouted following a different strategy is lower than 6 , which validates AFRO as a scheme performing network reoptimization with minimum traffic disruption.

Table 1. AFRO performance

\begin{tabular}{|c|c|c|c|c|c|c|c|c|c|c|c|c|c|c|}
\hline & \multicolumn{2}{|c|}{$\begin{array}{c}\text { Network } \\
\text { characteristics }\end{array}$} & \multicolumn{4}{|c|}{ AFRO results } & \multicolumn{3}{|c|}{$\begin{array}{l}\text { Avg. Link Load } \\
\text { (\# slices) }\end{array}$} & \multicolumn{3}{|c|}{$\begin{array}{l}\text { Max. Link Load } \\
\text { (\# slices) }\end{array}$} & \multicolumn{2}{|c|}{$\begin{array}{c}\text { Link } \\
\text { Entropy }\end{array}$} \\
\hline & $|N|$ & $|\boldsymbol{E}|$ & $|\boldsymbol{P}|$ & $\left|P^{\prime}\right|$ & $P * \mid$ & Max. Time & to & $t 2$ & $t 3$ & to & $t 2$ & $t 3$ & to & $t 3$ \\
\hline BT & 22 & 35 & 196 & 35 & 15 & $33.47 \mathrm{~s}$ & 163 & 169 & 163 & 445 & 503 & 454 & 3.9 & 3.86 \\
\hline TEL & 30 & 56 & 342 & 64 & 27 & $69.30 \mathrm{~s}$ & 198 & 204 & 196 & 485 & 544 & 491 & 3.4 & 3.35 \\
\hline
\end{tabular}

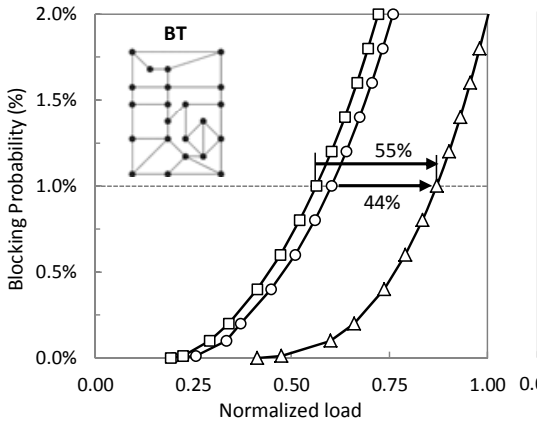

Fig. 4. Blocking probability vs. network load

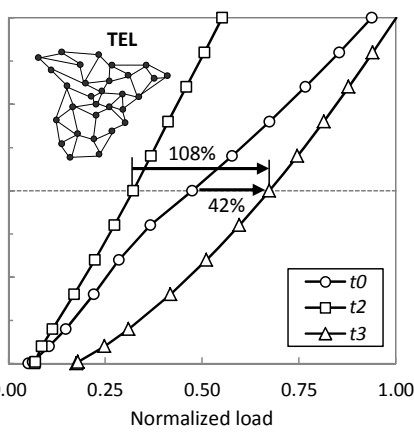

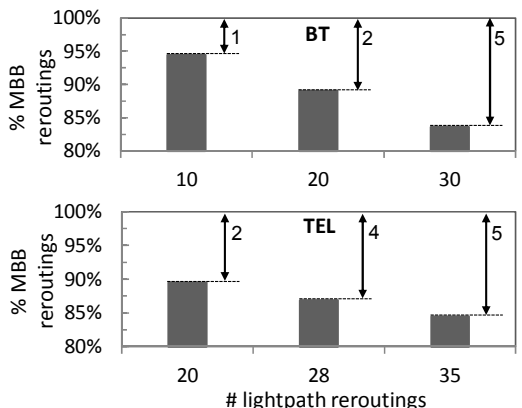

Fig. 5. Post-processing evaluation

\section{Conclusions}

The AFRO problem has been introduced as a network reoptimization problem with the objective of improving network capacity usage in dynamic flexgrid optical networks affected by a link failure. The obtained results for real networks show load gains between $42 \%$ and $108 \%$. Moreover, minimum traffic disruption is obtained when implementing changes in the network.

\section{References}

1. M. Jinno et al., "Spectrum-efficient and scalable elastic optical path network: architecture, benefits, and enabling technologies," IEEE Commun Mag, 47, 2009.

2. A. Castro, et al., "Experimental Demonstration of an Active Stateful PCE Performing Elastic Operations and Hitless Defragmentation," in Proc. ECOC, 2013.

3. A. Castro et al., "Dynamic Routing and Spectrum (Re)Allocation in Future Flexgrid Optical Networks," Comput Netw, 56, 2012.

4. A. Castro et al., "Dynamic Restoration in Multi-layer IP/MPLS-over-Flexgrid Networks," in Proc. DRCN, 2013.

5. P. Wright et al., "The Network Capacity Benefits of Flexgrid," in Proc. ONDM, 2013.

6. M. Ruiz et al., "Column Generation Algorithm for RSA Problems in Flexgrid Optical Networks," Photonic Netw Commun, 2013.

7. P. Wright et al., "Simulation Results of Shannon Entropy based Flexgrid Routing and Spectrum Assignment on a Real Network Topology", in Proc. ECOC, 2013. 